\title{
Numerical ability and improvement through interindividual cooperation varied between two cyprinid fish species, qingbo and crucian carp
}

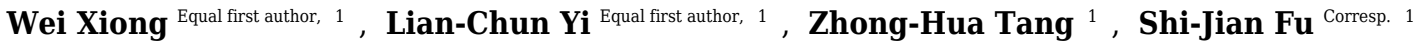 \\ ${ }^{1}$ Laboratory of Evolutionary Physiology and Behavior, Chongqing Key Laboratory of Animal Biology, Chongqing Normal University, Chongqing, China \\ Corresponding Author: Shi-Jian Fu \\ Email address: shijianfu9@cqnu.edu.cn
}

We used qingbo (Spinibarbus sinensis) and Chinese crucian carp (Carassius auratus) to test whether numerical discrimination could be improved by the coexistence and possible cooperation of conspecies or heterospecies. We conducted a spontaneous shoal choice test of singletons, conspecific dyads and heterospecific dyads under different numerical comparisons ( 8 vs. 12,9 vs. 12 and 10 vs. 12). Singletons of qingbo could discriminate only 8 vs. 12, whereas the dyads of qingbo showed better numerical acuity, as they could discriminate 10 vs. 12 . Crucian carp may have poor numerical ability, as both singleton and dyads showed no significant preference for larger stimulus shoals, even at the 'easier' numerical discrimination, i.e., 8 vs. 12 . Furthermore, heterospecific dyads of crucian carp and qingbo did not show significant preference for larger shoals at any numerical comparison in the present study. It is suggested that both the numerical ability and the possibility for improvement by interindividual interaction and hence cooperation might vary among fish species, and the interaction between heterospecies in the present study showed negative effect on numerical ability possibly due to the different behavioral and cognitive traits which make the information transfer and consensus difficult to reach. 
Numerical ability and improvement through

2 interindividual cooperation varied between two

3 cyprinid fish species, qingbo and crucian carp

4

5

6

7

Wei Xiong, Lian-Chun Yi, Zhong-Hua Tang, Shi-Jian Fu

Laboratory of Evolutionary Physiology and Behavior, Chongqing Key Laboratory of Animal

Biology, Chongqing Normal University, Chongqing, 401331, China

11

12

13

14

Corresponding Author:

Shi-Jian Fu

15 University Town, Shapingba District, Chongqing, 401331, China

Email address: shijianfu9@cqnu.edu.cn

17 


\section{Abstract}

19 We used qingbo (Spinibarbus sinensis) and Chinese crucian carp (Carassius auratus) to test

20 whether numerical discrimination could be improved by the coexistence and possible cooperation of conspecies or heterospecies. We conducted a spontaneous shoal choice test of

22 23 24 singletons, conspecific dyads and heterospecific dyads under different numerical comparisons (8 vs. 12,9 vs. 12 and 10 vs. 12). Singletons of qingbo could discriminate only 8 vs. 12 , whereas the dyads of qingbo showed better numerical acuity, as they could discriminate 10 vs. 12 . Crucian carp may have poor numerical ability, as both singleton and dyads showed no significant preference for larger stimulus shoals, even at the 'easier' numerical discrimination, i.e., 8 vs. 12 . Furthermore, heterospecific dyads of crucian carp and qingbo did not show significant preference for larger shoals at any numerical comparison in the present study. It is suggested that both the numerical ability and the possibility for improvement by interindividual interaction and hence cooperation might vary among fish species, and the interaction between heterospecies in the present study showed negative effect on numerical ability possibly due to the different behavioral and cognitive traits which make the information transfer and consensus difficult to reach.

\section{Introduction}

Many studies have demonstrated that the ability to discriminate between quantities is widespread (Vallortigara, 2012, 2017) and has been exhibited in preverbal infants (Lipton \& Spelke, 2003) as well as in a range of nonhuman animals, such as dolphins (Kilian et al., 2003), dogs (Ward and Smuts, 2007), chickens (Rugani, Regolin \& Vallortigara, 2007, 2008), tortoises (Gazzola, Vallortigara \& Pellitteri-Rose, 2018), frogs (Stancher et al., 2015), fish (Agrillo \& Dadda, 2007; Agrillo, Dadda \& Bisazza, 2007; Stancher et al., 2013; Miletto Petrazzini et al., 2015) and invertebrates (Gross et al., 2009; Reznikova \& Ryabko,2011). Numerical ability can improve antipredation efforts of animals by allowing them to compare shoals and join larger ones because larger shoals confer the ecological benefits of improved survivorship under the risk of predation (Foster \& Treherne, 1981; Landeau \& Terborgh, 1986;). Thus, it has been assumed that the ability to discriminate quantities and stay with a larger shoal is a vital advantage in fish species that live in groups. 
48 Improved cognitive ability through interindividual cooperation was suggested more than one 49 century ago (Galton, 1907), and the so-called 'collective intelligence' has frequently been proven 50 both in the field and in laboratory studies (Krause, Ruxton \& Krause, 2010; Bisazza et al., 2014).

51 The improvement in numerical ability has also been found in grass carp (Ctenopharyngodon 52 idellus) (Bai, Tang \& Fu, 2019) and guppy (Poecilia reticulata) (Bisazza et al., 2014). The 53 possible mechanisms might be due to the so-called 'meritocratic leadership', i.e., the 54 performance of a dyad is determined by the better member, which plays a leadership role in 55 decision making (Bisazza et al., 2014). In nature, mixed-species shoals are frequently found in a 56 wide range of animal taxa and provide advantages in terms of enhanced foraging efficiency and 57 predator avoidance (Goodale et al., 2010; Kleinhappel et al., 2016). Recently, using two cyprinid 58 fish species as experimental animals, we found that numerical ability might facilitate by 59 cooperation between mixed-species individuals of grass carp (Ctenopharyngodon idellus) (good numerical ability) and Chinese bream (Parabramis pekinensis) (poor numerical ability) as the latter exhibited shutter behavior between two stimulus shoals following those of grass carp and hence improved numerical ability compared to either singleton Chinese bream and its conspecific dyad (Bai, Tang \& Fu, 2019). Furthermore, the preference for either larger or smaller shoals might vary between singletons and dyads due to some emotional and (or) motivational factor (Regolin, Vallortigara \& Zanforlin, 1995). Thus, the aim of the present study was to test if the abovementioned improvement also manifests in other cyprinid fish species.

In the present study, we selected qingbo (Spinibarbus sinensis) and crucian carp (Carassius auratus) as the experimental animals. Both species prefer group living and are widely distributed throughout China. The numerical ability of qingbo (in singletons is 2:3) was recently documented (Xiong et al., 2018), while data on the numerical ability of crucian carp are unavailable. To fulfill our goal, we conducted a spontaneous shoal choice test of singletons and dyads (both conspecific and heterospecific) under a numerical comparison range of 8 vs. 12 to 10 vs. 12 ( 8 vs. 12 was used because almost all fish species showed a similar or higher numerical ability at a 2:3 ratio). We tested our hypothesis by comparing the preference to choose the larger stimulus shoal among singletons, conspecific and heterospecific dyads.

\section{Materials \& Methods}

\section{Ethics statement}


78

79

80

81

82

83

84

85

86

87

88

89

90

91

92

93

94

95

96

97

98

99

100

101

102

103

104

105

106

107

This study was approved by the Animal Care and Use Committee of the Key Laboratory of Animal Biology of Chongqing (Permit Number: Zhao-20161012-01) and was performed in strict accordance with the recommendations in the Guide for the Care and Use of Animal at the Key Laboratory of Animal Biology of Chongqing, China.

\section{Subjects and housing conditions}

Experimental juvenile qingbo $(\mathrm{N}=400$, standard body length $5.10 \pm 0.05 \mathrm{~cm}$; body mass 2.87 $\pm 0.09 \mathrm{~g})$ and juvenile crucian carp $(\mathrm{N}=400$, standard body length $3.88 \pm 0.04 \mathrm{~cm}$; body mass $1.74 \pm 0.07 \mathrm{~g}$ ) were purchased from a local market. Prior to the experiment, both qingbo and crucian carp were randomly divided into two groups and reared separately as either the stimulus shoal or the test fish. All fish were reared in water tanks that included a biological filter with recirculating water for four weeks. During this period, the temperature of the dechlorinated water was maintained at $25 \pm 1{ }^{\circ} \mathrm{C}$, and the oxygen content was maintained above $90 \%$ saturation. The photoperiod cycle was $12 \mathrm{~h}$ of light: $12 \mathrm{~h}$ of darkness. All fish were fed once daily at 8:00 am with a commercial floating diet (Tongwei Company, China). The uneaten food and feces were cleaned up using a siphon. Furthermore, the experimental measurements were performed between 8:00 and 18:00 after four weeks of acclimation.

\section{Experimental design}

We conducted 360 spontaneous choice tests, i.e., 20 repetitions of choice tests for singletons of qingbo, singletons of crucian carp, dyads of qingbo, dyads of crucian carp and dyads of mixed-species pairs (one qingbo and one crucian carp). Either a single (singleton test) or a pair of fish (dyad test) was used as the test fish for each choice test. The numerical comparison was 8 vs. 12,9 vs. 12 and 10 vs. 12 .

\section{Protocol and setup}

The experimental apparatus used to assess the spontaneous shoal choice test consisted of a test tank $(70 \mathrm{~cm} \times 35 \mathrm{~cm} \times 35 \mathrm{~cm})$ with two stimulus tanks $(20 \mathrm{~cm} \times 35 \mathrm{~cm} \times 35 \mathrm{~cm})$ positioned at both ends (Fig. 1). Two 16-watt fluorescent lamps were located at both ends of the stimulus tank. The tanks were filled with $10 \mathrm{~cm}$ of water. To prevent other interference factors, all of the walls of the test tank, except for the wall connected with the stimuli tank, were covered with white paper, and an opaque curtain was stretched around the arena. During the test, the behavioral responses of the test fish were recorded using a webcam (Logitech Pro 9000; Logitech Company, 
108 Suzhou, China) located $100 \mathrm{~cm}$ over the test tank and above the arena and connected to a remote 109 computer..

110 To avoid foraging behavior, fish were fed to apparent satiation $2 \mathrm{~h}$ before the measurements.

111 Stimulus fish were first introduced to the stimulus tank $5 \mathrm{~min}$ prior to the test. The test fish were 112 then introduced to the center of the test tank with a holding device (plastic cylinder with $10 \mathrm{~cm}$ 113 of diameter and $15 \mathrm{~cm}$ of height) and remained in the device for $3 \mathrm{~min}$. After that period, the 114 holding device was gently removed, and the position of the test fish was recorded for $15 \mathrm{~min}$ at 11515 frames s$^{-1}$. Each individual test fish was used only once, whereas individuals of the stimulus 116 shoals may have been used more than once. Twenty repetitions of each treatment were recorded. 117 In the numerical preference assessmentfor singletons or conspecific dyads, all stimulus shoal 118 members were the same fish species. In the numerical preference assessmentfor heterospecific 119 dyads, half qingbo and half Chinese crucian carp formed a mix stimulus shoals of two species for 1208 vs. 12 and 10 vs. 12 numerical comparison, whereas 10 groups of 5 qingbo mixed with 4 121 Chinese crucian carp and 10 groups of 4 qingbo mixed with 5 Chinese crucian carp for stimulus 122 shoal of 9 individuals. The positions of the test fish were analyzed by the automated tracking 123 software program id Tracker (v 2.1) (Pérez-Escudero et al., 2014; Tang et al., 2017). In 124 conspecific dyads, the video files of two of the measurements of 8 vs. 12 in qingbo, three of the 125 measurements of 9 vs. 12 in crucian carp and one measurement of 10 vs. 12 in crucian carp 126 failed to be decoded. Therefore, the final repetitions were 18, 17 and 19 in 8 vs. 12, 9 vs. 12 and 12710 vs. 12, respectively. The preferences of the test fish were defined as the time spent by the test 128 fish within the $11 \mathrm{~cm}$ preference zones during the 15-min periods of recording (i.e., within $11 \mathrm{~cm}$ 129 of the wall adjacent to the stimulus tanks on either side) and were calculated as follows:

130 observations within the preference zone of either the larger or smaller shoal / (observations 131 within the preference zone of the larger shoal + observations within the preference zone of the 132 smaller shoal). In conspecific dyads, we measured each fish in the dyad independently and then 133 calculated the average for dyads.

\section{Statistical analysis}

135 Statistical tests were carried out using SPSS 11.5. The time spent in the preference zone was 136 recorded as a measure of the preference of each test fish for either the larger or smaller stimulus 137 shoal. All data were tested for normality by a one-sample Kolmogorov-Smirnov test. The 138 linearly mixed model (tested number as random factor) was used to test the significance of the 
139 preference for larger or smaller shoals. All values are presented as the mean \pm S.E., and $P<0.05$ 140 was used as the level of statistical significance.

\section{Results}

142 Experiment 1 Numerical ability of singletons

143 Qingbo showed a significant preference for larger shoals at only a numerical contrast of 8 vs. $14412\left(F_{1,38}=6.083, P=0.018\right)$, i.e., qingbo were able to discriminate at only a 2:3 ratio (Fig. 2).

145 No significant preference was observed for 9 vs. $12\left(F_{1,38}=0.119, P=0.732\right)$, or 10 vs. $12\left(F_{1,38}\right.$ $146=1.742, P=0.195)$. Crucian carp showed no significant preference for larger shoals at any 147 numerical comparison: 8 vs. $12\left(F_{1,38}=0.223, P=0.640\right), 9$ vs. $12\left(F_{1,38}=3.741, P=0.061\right)$, or 14810 vs. $12\left(F_{1,38}=0.335, P=0.567\right)$.

\section{Experiment 2 Numerical ability of conspecific dyads}

150 Dyads of qingbo showed a significant preference for the larger shoal at numerical contrasts of 1518 vs. $12\left(F_{1,34}=7.874, P=0.008\right)$ and 10 vs. $12\left(F_{1,38}=4.581, P=0.039\right)$ but not at 9 vs. 12 $152\left(F_{1,38}=2.662, P=0.111\right)$. However, no significant preference was observed at any numerical 153 comparison in crucian carp: 8 vs. $12\left(F_{1,38}=0.235, P=0.631\right), 9$ vs. $12\left(F_{1,32}=0.064, P=\right.$ $1540.801)$, or 10 vs. $12\left(F_{1,36}=0.511, P=0.479\right)$.

\section{Experiment 3 Numerical ability of heterospecific dyads}

156 Neither qingbo nor crucian carp showed any significant preference for larger shoals at any 157 numerical comparison in the preset study; 8 vs. 12 (qingbo: $F_{1,38}=0.818, P=0.371$, crucian 158 carp: $\left.F_{1,38}=0.704, P=0.407\right), 9$ vs. $12\left(F_{1,38}=2.118, P=0.154, F_{1,38}=0.969, P=0.331\right)$, and 15910 vs. $12\left(F_{1,38}=0.297, P=0.589, F_{1,38}=0.155, P=0.696\right)$.

160 Discussion

161 The main aims of the present study were to test whether interaction (i.e., cooperation) between 162 individuals could facilitate the numerical abilities of two cyprinid fish species and whether the interaction between different fish species could achieve similar performance compared to the possible improvement between conspecific dyads. The numerical ability indicated by shoal preference increased from a threshold of 8 vs. 12 in singleton qingbo to at least $10 \mathrm{vs}$. 12 (the most subtle numerical difference in this study) in qingbo dyads. However, crucian carp may show poor numerical ability as it showed no preference to larger shoal in any numerical comparison in the present study. Thus, the possible improvement in numerical ability between individual crucian carp and heterospecific dyads was difficult to assess; however, the 
170

171

172

173

174

175

176

177

178

179

180

181

182

183

184

185

186

187

188

189

190

191

192

193

194

195

196

197

198

199

200

coexistence of heterospecies did show a negative effect on the numerical ability of qingbo, as the qingbo in heterospecific dyads could not even discriminate in the numerical comparison of 8 vs. 12 .

\section{Qingbo showed greater numerical ability indicated by shoal preference than crucian carp}

Given the ecological benefit of collective living, half of all fish species prefer group living throughout life or for at least a part of their life history (Shaw, 1978). Thus, numerical discrimination may play an important role in fish species (Miletto Petrazzini et al., 2015), and such ability has been widely documented in fish species (Agrillo, Dadda \& Bisazza, 2007; Potrich et al., 2015; Xiong et al., 2018). In the present study, singletons of qingbo could discriminate in the numerical comparison of 8 vs. 12, which fall within the data of other fish species (Lucon-Xiccato \& Bissazza, 2017), which usually varied from 0.5 to 0.75 ). However, crucian carp showed no significant preference for larger shoals in any of the numerical comparisons in the present study. It is suggested that differences in numerical ability might exist in closely related fish species. This may be reasonable, as qingbo in the field prefer to live in fast-flowing water and exhibit a group-living lifestyle (Killen et al., 2016; Tang et al., 2017). However, crucian carp prefer to live in still water with a more complicated habitat, and selection stress may be more severe on characteristics other than group living and numerical ability. However, cognitive and behavioral traits may also be involved in such differences in the artificial experimental conditions, as the crucian carp seemed much less bold and showed fewer exploration activities than qingbo. According to our observation (not directly recorded), it seemed that Chinese crucian carp performed considerably fewer shutters between two stimulus shoals than qingbo in the present study. Further investigation on boldness and stress hormone such as plasma cortisol level during choice test might provide useful information. Furthermore, the data in Fig. 2 clearly show that the mean preference ratio of crucian carp singletons, under the numerical comparison of 8 vs. 12 (possibly also under the 8 vs. 12 and 9 vs. 12 conspecific dyads), was similar to that of the qingbo. It is suggested that the large interindividual variation might mask the possible shoal preference of crucian carp under these situations. The reason might be that some individuals were rather shy and would stay with the smaller stimulus shoal once they made the wrong choice (Lucon-Xiccato et al., 2017). Therefore, we cannot tell if they cannot discriminate in the numerical comparison, if the test fish dare not make a choice or if they do not care about the difference under the conditions of the present study. Perhaps a further study 
201 under a food choice test might provide more useful information, as a previous study found that

202 the numerical ability varied between different ecological contexts (Lucon-Xiccato and Dadda, 203 2017). Nevertheless, the present study suggested that the numerical discrimination threshold of 204 singleton qingbo is approximately 2:3, whereas the threshold of crucian carp is less than that if it 205 has any numerical ability or shoal preference.

\section{The dyads of qingbo showed better numerical acuity than sigletons}

207 Increased cognitive ability through interindividual interaction was again demonstrated in the 208 present study, as the numerical threshold of qingbo dyads improved profoundly when compared 209 to that of singletons. The previous studies in grass carp (Bai et al., 2019) and guppy (Bisazza et

210 al., 2014) found both species can discriminate at a ratio of 0.75 (i.e., 3:4) by interindividual 211 cooperation. In the present study, dyads of qingbo could even discriminate in the 10 vs. 12 212 comparison (i.e., 0.833). Given that it is the smallest numerical comparison treatment designed in

213 the present study, it is suggested that qingbo may achieve a considerably high numerical 214 discrimination performance through cooperation. However, it was worth noting that dyads of 215 qingbo fail to discriminate 9 vs. 12, even though the preference for larger stimulus shoals was $23 \%$ 216 higher than that for smaller shoals. It is possible that the aforementioned large interindividual 217 variation might mask the significant difference. The improvement in numerical ability by 218 interindividual cooperation has also been found in grass carp (Ctenopharyngodon idellus) (Bai, 219 Tang \& Fu, 2019). Given the profound improvement in numerical ability, it is possible that the 220 'meritocratic leadership' might be involved in cooperation during the numerical discrimination

221 process. However, it has long been suggested that the emotional and (or) motivational factor 222 related to the numerical discrimination and spatial cognition might also be involved in the 223 improved performance between dyads and singletons (Regolin, Vallortigara \& Zanforlin, 1995).

Both singletons and dyads of crucian carp showed no shoal preference at any shoal preference 225 in the present study; thus, we cannot draw any conclusions about the possibility for improvement 226 in numerical ability by the interindividual interaction of crucian carp. However, because qingbo 227 could improve from the numerical comparison of 8 vs. 12 in singleton to $10 \mathrm{vs}$. 12 in dyad, if 228 such improvement does exist in crucian carp, the threshold of singleton crucian carp must be 229 considerably lower than 7 vs. 12 (which is the best possible numerical contrast threshold of the 230 crucian carp dyad). Again, the spontaneous shoal choice test under another ecological context 
231 (for example, mating or foraging) or a training protocol used before might provide more useful 232 information (Lucon-Xiccato and Bisazza, 2017).

233 Qingbo in heterospecific dyads showed poor numerical ability even lower than singletons

234 In the present study, the crucian carp in the heterospecific dyads showed no shoal preference 235 in any numerical comparisons. However, because we did not conduct an easier numerical 236 comparison than 2:-3, we cannot determine the thresholds of singleton or dyads of crucian carp, 237 if they have any. Thus, we also cannot draw any conclusions about the possibility of the 238 numerical ability (or shoal preference) of crucian carp increasing through heterospecific 239 cooperation. However, on the contrary, the company of crucian carp showed a negative effect on 240 the numerical ability of qingbo, as qingbo in heterospecific dyads could not even discriminate in 241 the 8 vs. 12 comparisons (the threshold of singletons). These mechanisms need further 242 investigation. Because these two fish prefer living in different habitats, the behavioral and 243 cognitive traits might be completely different, thus making the information transfer and 244 consensus difficult to reach, which may be the precondition of the collective intelligence. Further 245 246 investigation using fish species of naturally mixed shoals as experimental models might give more useful information.

247

248

\section{Conclusions}

249 Qingbo showed a similar numerical acuity to previous studies with a threshold of 0.66 , which 250 could be facilitated to at least 0.83 by interindividual cooperation. Crucian carp showed no shoal 251 preference in the present study, possibly due to low cognitive ability, large interindividual variation in numerical ability, low boldness or no motivation for shoal preference, or improper 253 numerical comparison (i.e. only large numerical comparison), which might make this a poor experimental model for numerical ability research. The interaction between heterospecific dyads elicited a decreased numerical ability of qingbo, which became even lower than the threshold of singletons. However, it is difficult to draw any safe conclusions about the difference in cooperation efficiency on numerical comparisons between conspecific and heterospecific dyads due to the poor performance of crucian carp and (or) lacking of small numerical comparison in the present study.

260 Acknowledgments 
261 We would like to thank Dr. Jennifer Vonk and two anonymous reviewers for insightful

262

263

264

265

266

267

268

269

270

271

272

273

274

275

276

277

278

279

280

281

282

283

284

285

286

287

288

289

290

291

292

293

294

295

296

297

298

299

300

301

302

303

304

comments and constructive suggestions that greatly improved the manuscript.

\section{References}

Agrillo C, Dadda M, Bisazza A. 2007. Quantity discrimination in female mosquitofish. Animal Cognition 10:63-70

Agrillo C, Dadda M. 2007. Discrimination of the larger shoal in the poeciliid fish Girardinus falcatus. Ethology Ecology and Evolution 19:145-157

Bai Y, Tang ZH, Fu SJ. 2019. Numerical ability in fish species: preference between shoals of different sizeds varies among signletons, conspecific dyads andheterospecific dyads. Animal Cognition. Online early

Bisazza A, Butterworth B, Piffer L, Bahrami B, Miletto Petrazzini ME, Agrillo C. 2014. Collective enhancement of numerical acuity by meritocratic leadership in fish. Scientific Reports 4:514-521

Foster WA, Treherne JE. 1981. Evidence for the dilution effect in the selfish herd from fish predation on a marine insect. Nature 293:466-467

Galton F. 1907. Vox populi. Nature 75:450-451

Gazzola A, Vallortigara G, Pellitteri-Rosa D. 2018. Continuous and discrete quantity discrimination in tortoises. Biology Letter 14: 20180649

Goodale E, Beauchamp G, Magrath RD, Nieh JC, Ruxton GD. 2010. Interspecific information transfer influences animal community structure. Trends in Ecology and Evolution 25:354361

Gross HJ, Pahl M, Si A, Zhu H, Tautz J, Zhang S. 2009. Number-based visual generalization in the honeybee. PLoSONE 4:e4263

Kilian A. Yaman S, von Fersen L, Gunturkun O. 2003. A bottlenose dolphin discriminates visual stimuli differing in numerosity. Learn Behavior 31:133-142

Killen SS, Fu C, Wu Q, Wang YX Fu SJ. 2016. The relationship between metabolic rate and sociability is altered by food deprivation. Functional Ecology 30:1358-1365

Kleinhappel TK, Burman OHP, John EA, Wilkinson A, Pike TW. 2016. A mechanism mediating inter-individual associations in mixed-species groups. Behavioral Ecology and Sociobiology 70:755-760

Krause J, Ruxton GD, Krause S. 2010. Swarm intelligence in animals and humams. Trends in Ecology and Evolution 25:28-34

Landeau L, Terborgh J. 1986. Oddity and the 'confusion effect' in predation. Animal Behavior 34:1372-1380

Lipton JS, Spelke E. 2003. Origins of number sense: large-number discrimination in human infants. Psychological Science 14: 396-401

Lucon-Xiccato T, Bisazza A. 2017. Individual differences in cognition among teleost fishes. Behavior Processes 141:184-195

Lucon-Xiccato T, Dadda M. 2017. Individual guppies differ in quantity discrimination performance across antipredator and foraging contexts. Behavioral Ecology and Sociobiology 71: 13

Lucon-Xiccato T, Dadda M, Gatto E, Bisazza A. 2017. Development and testing of a rapid method for measuring shoal size discrimination. Animal Cognition 20:149-157 
305

306

307

308

309

310

311

312

313

314

315

316

317

318

319

320

321

322

323

324

325

326

327

328

329

330

331

332

333

334

335

336

337

338

Miletto Petrazzini M E, Lucon-Xiccato T, Agrillo C, Bisazza A. 2015. Use of ordinal information by fish. Scientific Reports 5:15497

Pérez-Escudero A,Vicente-Page J, Hinz RC, Arganda S, DePolavieja GG. 2014. idTracker: tracking individuals in a group by automatic identification of unmarked animals. Nature Methods 11:743-748

Potrich D, Sovrano VA, Stancher G., Vallortigara G. 2015. Quantity discrimination by zebrafish (Danio rerio). Journal of Comparative Psychology 129: 388-393

Regolin L, Vallortigara G, Zanforlin M. 1995. Detour behaviour in the domestic chick: searching for a disappearing prey or a disappearing social partner. Animal Behaviour 50:203-211.

Reznikova Z, Ryabko B. 2011. Numerical competence in animals, with an insight from ants. Behaviour 148:405-434

Rugani R, Regolin L, Vallortigara G. 2008. Discrimination of small numerosities in young chicks. Journal of Experimental Psychology: Animal Behavior Processes 34:388-399

Rugani R, Regolin L, Vallortigara G. 2007. Rudimental numerical competence in 5-day-old domestic chicks (Gallus gallus): Identification of ordinal position. Journal of Experimental Psychology: Animal Behavior Processes 33: 1-31

Shaw E. 1978. Schooling fishes. American Scientist 66:166-175

Stancher G, Rugani R, Vallortigara G. 2015. Numerical discrimination by frogs (Bombina orientalis). Animal Cognition 18: 219-2209

Stancher G, Sovrano VA, Potrich D, Vallortigara G. 2013. Discrimination of small quantities by fish (redtail splitfin Xenotoca eiseni). Animal Cognition 16: 307-312

Tang ZH, Wu H, Huang Q, Kuang L, Fu SJ. 2017. The shoaling behavior of two cyprinid species in conspecific and heterospecifi groups. PeerJ 5:e3397

Vallortigara G. 2012. Core knowledge of object, number, and geometry: A comparative and neural approach, Cognitive Neuropsychology 29: 213-236

Vallortigara G. 2017. Comparative cognition of number and space: the case of geometry and of the mental number line. Philosophical Transactions of the Royal Society B 373: 20170120 .

Ward C, Smuts B. 2007. Quantity based judgments in the domestic dog (Canis lupusfamiliaris). Animal Cognition 10:7 1-80

Xiong W, Yi LC, Tang ZH, Zhao X, Fu SJ. 2018. Quantity discrimination in fish species: fish use non-numerical continuous quantity traits to select shoals. Animal Cognition 21: 813-820 
Figure 1

Experimental setup showing the structure of experimental arena.

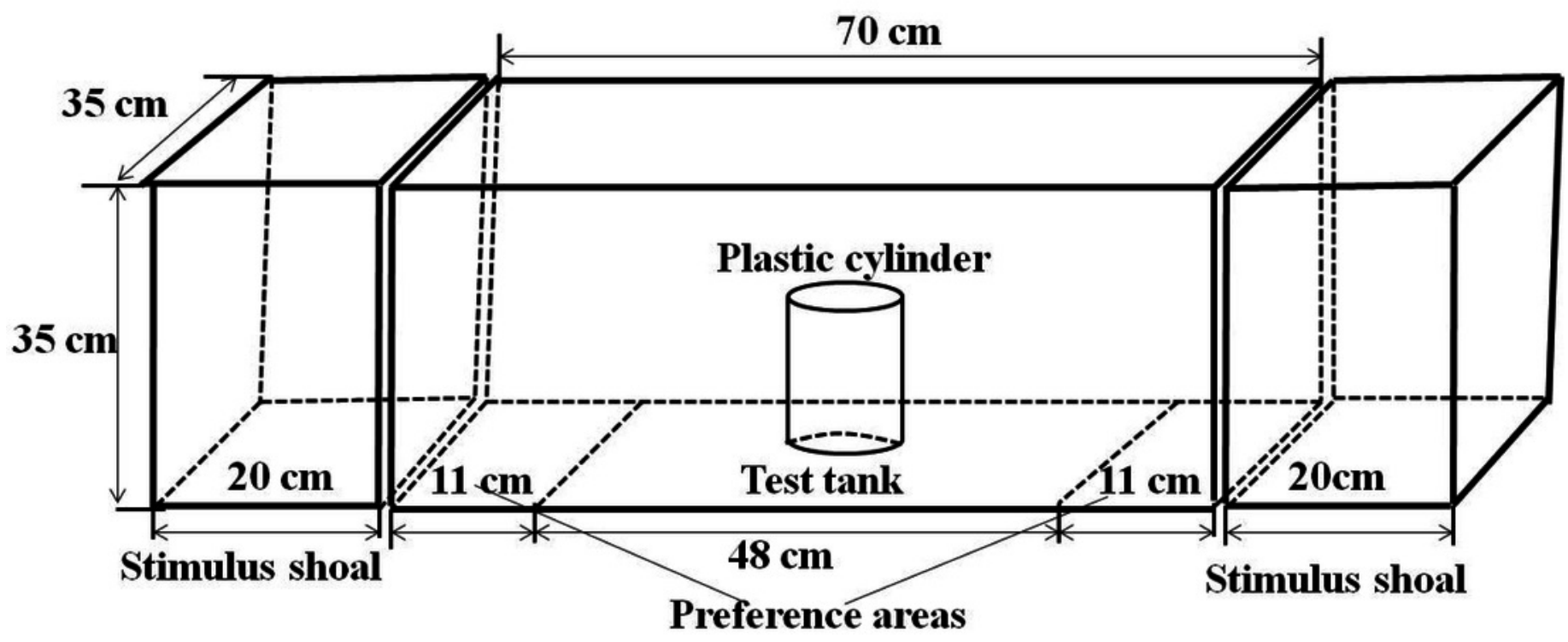




\section{Figure 2 (on next page)}

The shoal choice test of qingbo and crucian carp in both singletons and dyads (both conspecific and heterospecific) under different numerical comparisons

Mean \pm S.E., 20 repetitions, except $N=18$ for 8 vs. 12 of conspecific dyad of qingbo, 17 for 9 vs. 12 and 19 for 10 vs. 12 of conspecific dyads of crucian carp. $*$ indicates significant preference for larger shoals $(P<$ $0.05)$. 


\section{Manuscript to be reviewed}

A) Singletons

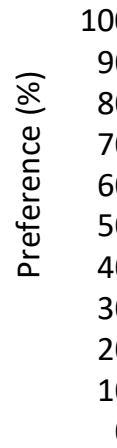

$\square$ Time near larger shoal

$\square$ Time near smaller shoal

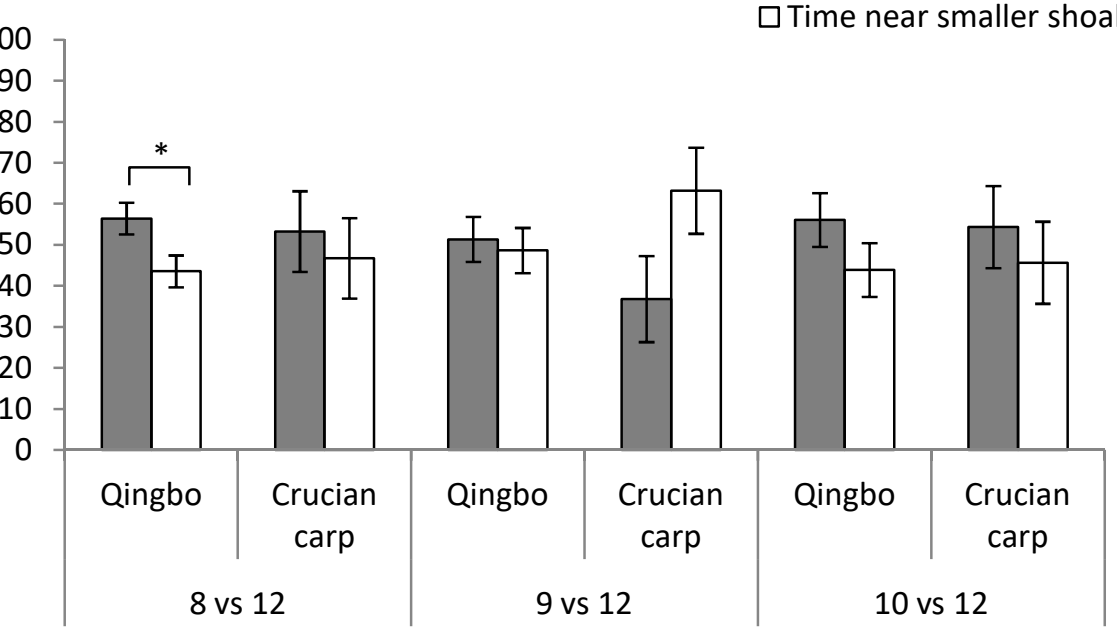

Numerical comparison

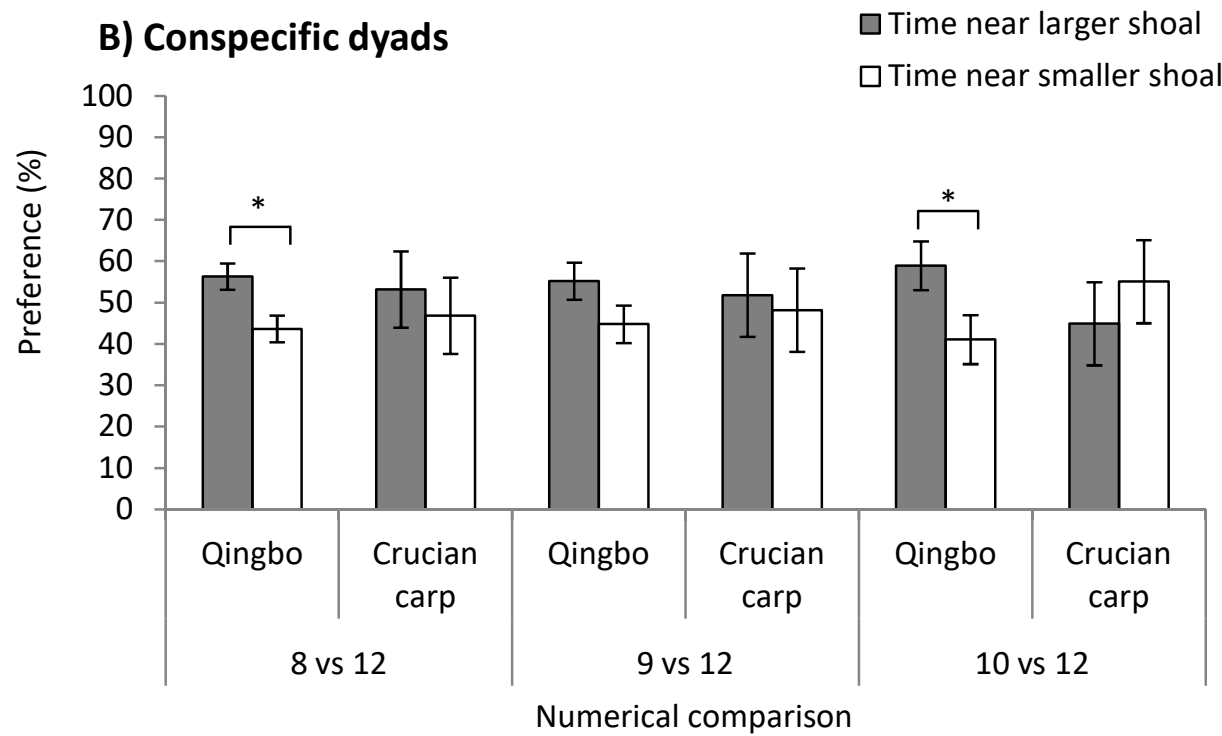

C) Heterospecific dyads

$\square$ Time near larger shoal

$\square$ Time near smaller shoal

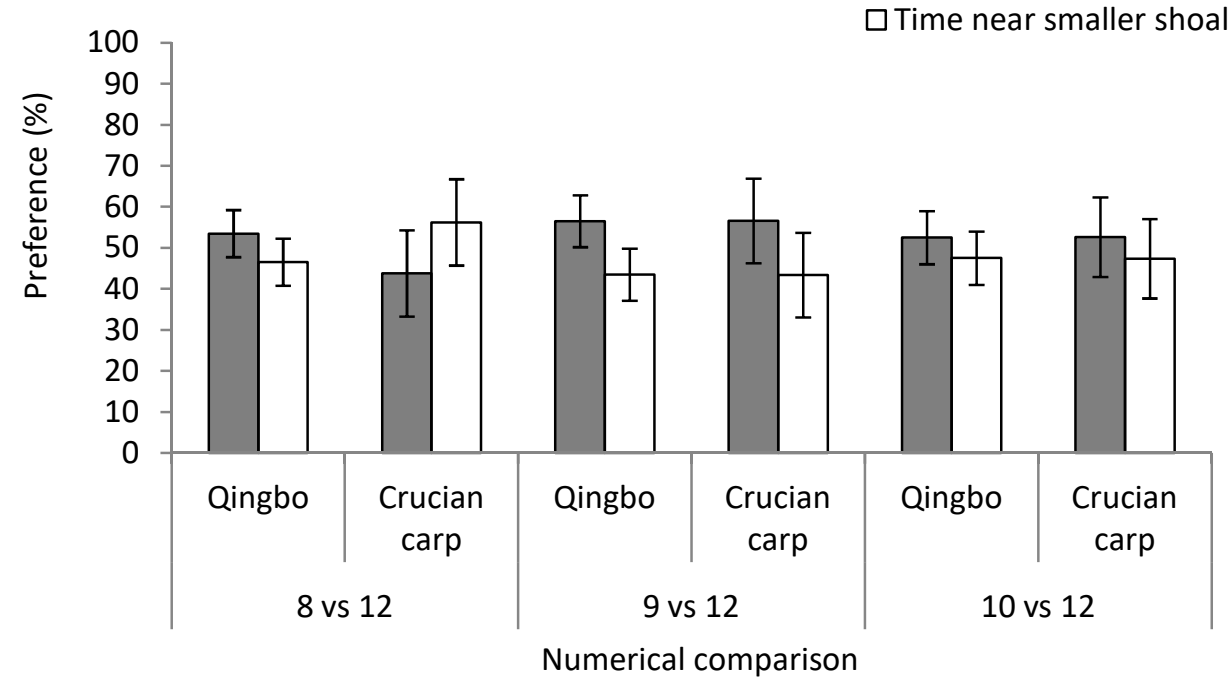

